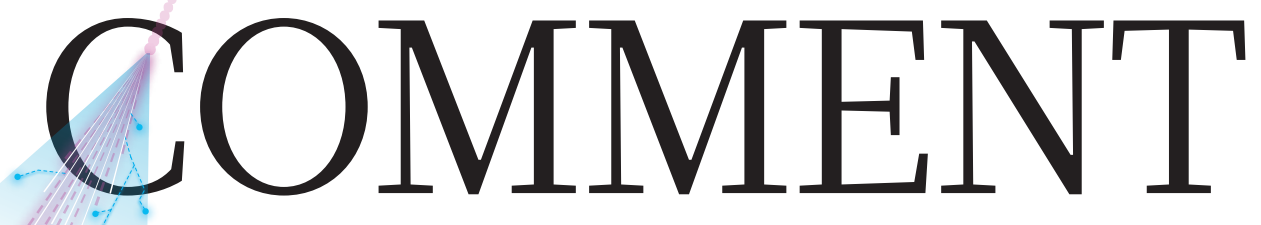

PHYSICS Which nextgeneration neutrino facility should be funded? p.462
ARCHAEOLOGY Sea-bed trove from Ancient Egypt's longsunk cities on show $\mathbf{p . 4 6 6}$
HEALTH A memoir of a life leading the fight against smallpox and HIV p.468
OBITUARY Harry Kroto, buckyball co-discoverer, remembered $\mathbf{p . 4 7 0}$

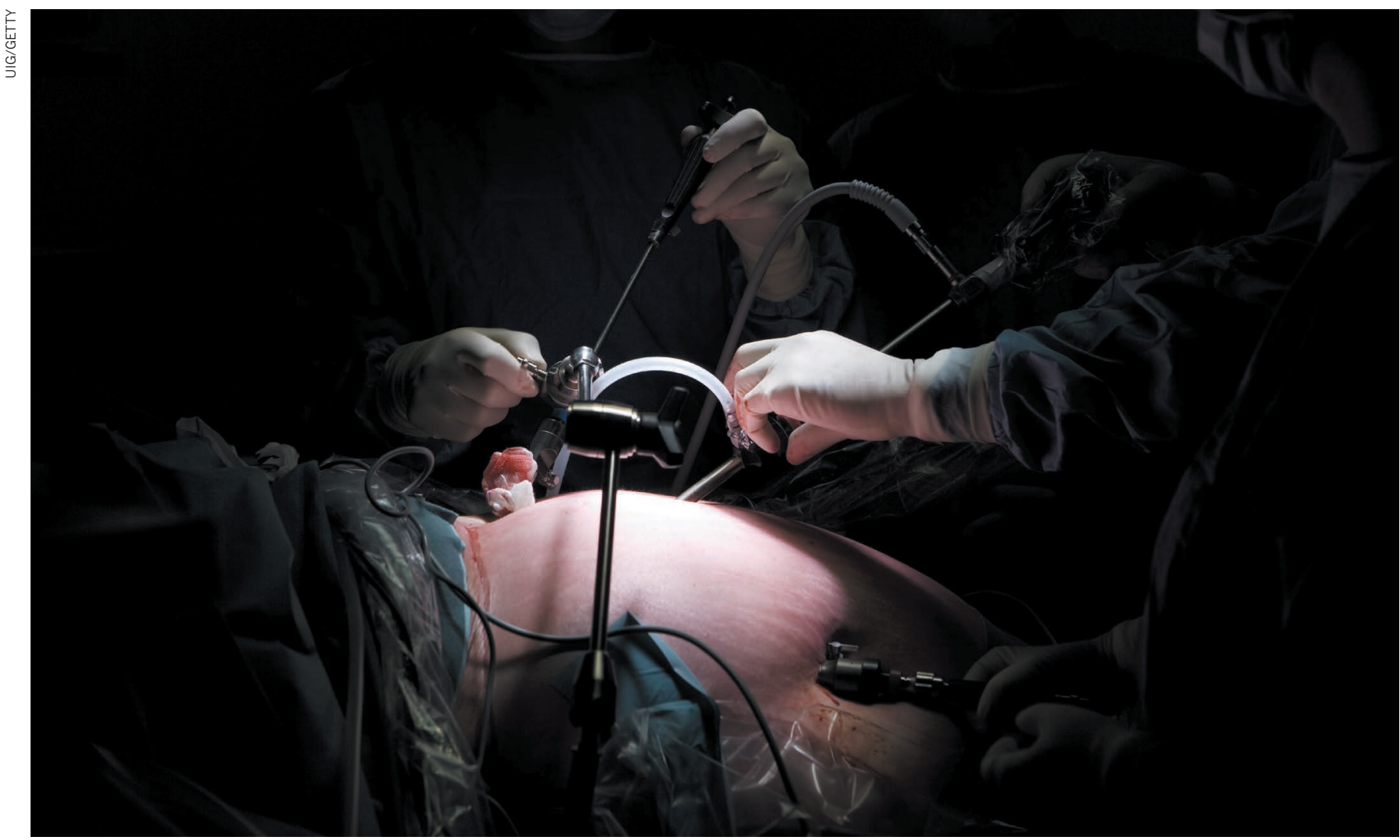

Surgery can be an effective treatment for type 2 diabetes.

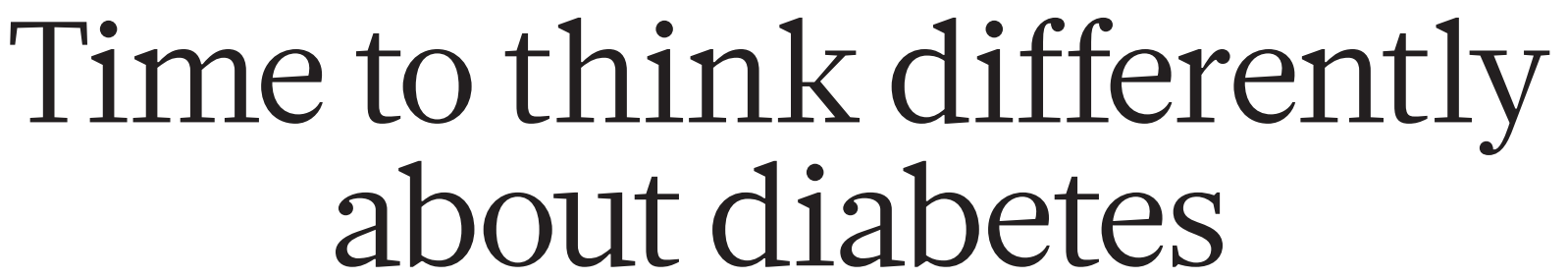

New guidelines for the surgical treatment of type 2 diabetes bolster hopes of finding a cure, writes Francesco Rubino, but long-standing preconceptions must be put aside.

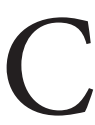

linical guidelines published this week $^{1}$ announce what may be the of type 2 diabetes for almost a century. Appearing in Diabetes Care, a journal of the American Diabetes Association, and endorsed by 45 professional societies around the world, the guidelines propose that surgery involving the manipulation of the stomach or intestine be considered as a standard treatment option for appropriate candidates. This development follows multiple clinical trials showing that gastrointestinal surgery can improve blood-sugar levels more effectively than any lifestyle or pharmaceutical intervention, and even lead to long-term remission of the disease ${ }^{1}$.

As someone who has been investigating the link between gastrointestinal surgery and glucose homeostasis since the late 1990s (see 'Surgical breakthrough'), I have witnessed first-hand how getting to this point has required many clinical scientists to put aside long-standing preconceptions. Indeed, the guidelines come nearly 100 years after the first clinical observations that diabetes could be improved or even resolved by a surgical operation (see 'A long road') ${ }^{2}$. The evidence that surgery can prompt the remission of a disease that has long been considered $>$ 


\section{DIABETES TREATMENT}

\section{Surgical breakthrough}

In 1925, a report in The Lancet ${ }^{2}$ described a 'side effect' of a gastrointestinal operation to treat a peptic ulcer. This was the almost overnight resolution of an excess of sugar in the urine (glycosuria) — the chief symptom of diabetes at the time. Similar observations were reported in subsequent decades and became more common after the advent of bariatric or weightloss surgery in the mid 1950s, which led to more people with diabetes receiving these types of operations. And during the 1980 s and 1990s, resolution of diabetes after bariatric surgery was noted on many occasions, including in a landmark report involving more than 120 patients ${ }^{9}$.

In 1999, while working as a research fellow at Mount Sinai School of Medicine in New York City, I stumbled across a report showing that nearly all people with type 2 diabetes who had undergone a complex bariatric operation (biliopancreatic diversion) had completely normal bloodsugar levels as early as one month after surgery. They had been able to stop taking medication and come off a low-calorie diet. I wondered whether gastrointestinal surgery could influence diabetes directly. If so, surgery could be used to treat diabetes or to understand how it works.

The next day, I persuaded my mentor to seek approval from the institutional review board to run trials in humans. Failing to obtain approval, we turned to rats to investigate whether a modified form of gastric-bypass surgery could directly influence glucose homeostasis. Our experiments confirmed that it could, although it took us more than two years to publish the findings ${ }^{6}$.

In 2006 and 2007, surgical teams showed that the operation had the same effect in humans ${ }^{10}$, and other groups began to investigate the molecular mechanisms that might be responsible. On the back of these studies, a multidisciplinary group of leading clinicians and scientists at the first Diabetes Surgery Summit in 2007 reviewed the preliminary mechanistic and clinical data available on the effects of surgery on diabetes and established an agenda for research priorities. The summit inspired the randomized clinical trials that now provide the evidence supporting a role of surgery in diabetes. In September 2015, the introduction of surgery into standard care for type 2 diabetes was formally recommended by the participants of the second Diabetes Surgery Summit ${ }^{1}$. F.R. irreversible could bolster searches for what causes diabetes and even reinvigorate hopes to find a cure. But future progress will require more thinking outside the box.

\section{CLINICAL SHIFT}

The number of adults around the world with diabetes quadrupled from 108 million in 1980 to 422 million in 2014 (ref. 3). About $90 \%$ of these people have type 2 diabetes a major cause of kidney failure, blindness, nerve damage, amputations, heart attack and stroke. Fewer than $50 \%$ of people with type 2 diabetes control their blood-sugar levels adequately by changing their diet or exercise regime, or by taking drugs.

Bariatric or weight-loss surgery refers to various procedures. Surgeons may, for instance, remove a portion of the person's stomach or divide the stomach into two and reroute the small intestine to the upper part (see 'Gastric bypass'). Since the mid 1950s, people whose body mass index (BMI) is greater than 40 have received bariatric surgery to induce weight loss. Many of these people also had diabetes. The new guidelines advise that such procedures (metabolic surgery) be considered specifically for the treatment of diabetes in people who have not adequately controlled their blood-sugar levels through other means, and whose BMI is greater than 30 (or 27.5 for people of Asian descent). Perhaps more significantly, they also state that the gastrointestinal tract is an appropriate biological target for interventions designed to treat diabetes ${ }^{1}$.

These recommendations arguably signify the most radical departure from mainstream approaches to the management of diabetes since the introduction of insulin in the 1920s. They are based on findings from a large body of work, including 11 randomized clinical trials conducted over the past decade ${ }^{1}$. In these studies, most

\section{BIG BENEFITS}

In one clinical trial, most people with diabetes who had received gastroinstestinal surgery did not need to take insulin or other injectable medication to control their blood-sugar levels even five years after the operation.

Not undergoing surgery $\square$ Undergoing surgery

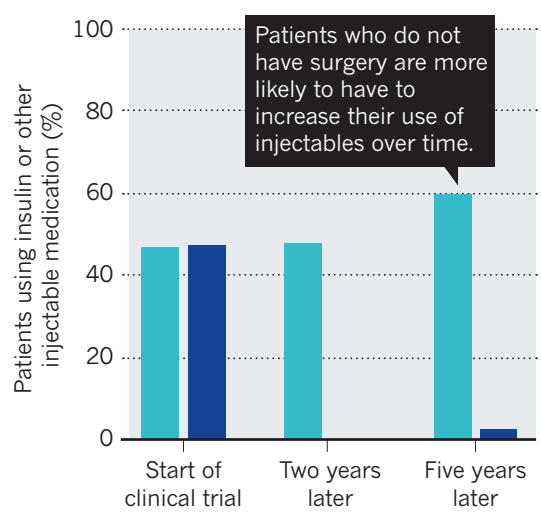

surgically treated people (up to $80 \%$ in a recent 5-year follow-up ${ }^{4}$ of a randomized trial) fall into one of two categories. Either their diabetes goes into apparent remission or their blood-sugar levels can be stabilized using reduced medication or exercise and a calorie-controlled diet (see 'Big benefits').

Non-randomized studies, involving people receiving surgery and matched subjects treated with standard interventions, suggest that surgery may also reduce heart attacks, stroke and diabetes-related mortality ${ }^{1}$. And several economic analyses suggest that the costs of surgery (roughly US\$20,000-25,000 per procedure in the United States) may be recouped within 2 years through reduced spending on medication and care ${ }^{5}$.

The effects of surgery on diabetes are dramatic. Yet it has taken nearly a century to unearth them since observations of major improvement or remission of diabetes after surgical operations were first reported ${ }^{2}$.

A major stumbling block seems to have been the lack of a plausible mechanism to explain how gastrointestinal surgery is able to resolve the symptoms of diabetes. Numerous surgeries - knee and hip replacements, appendix removal, even bariatric surgery - have been performed for decades without randomized trials confirming that these approaches are more effective than less invasive ones. But surgery explicitly seems to fix what is broken in those instances. However, in the case of diabetes - a systemic disease with dysfunctions involving the pancreas, liver, muscle and fat (adipose) tissue - it has been much harder to imagine what surgery would be able to mend.

The dominant 'adipocentric model' has also been a major conceptual barrier to the acceptance of surgery as a treatment for the disease itself. This model posits that excess fat causes diabetes, either by causing the liver to malfunction or by making other cells resistant to insulin. Because this model predicts that the reduction of fatty tissue, however obtained, can relieve the symptoms of diabetes, weight loss after bariatric surgery has provided a straightforward explanation for the associated remission of the disease.

It was exactly this absence of understanding about mechanism - and the mismatch between observations and mainstream thinking - that delayed the prescription of the painkiller aspirin to people with heart disease in the twentieth century. Clinical observations in the early 1950s suggested that aspirin could prevent thromboses. But large-scale trials to test the drug's ability to prevent heart attacks began only in the 1970s, after experiments had shown that it could inhibit blood clotting.

We now know that the dramatic effects of surgery on diabetes are not just a consequence of weight loss. Changes to gastrointestinal 
A LONG ROAD

Observations that diabetes can be improved or even resolved by surgical operations have been reported for almost a century.

1930

1940 1950

1955

Weight-loss

(bariatric) sugery

introduced.

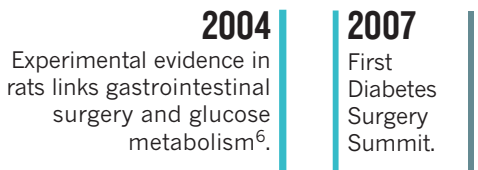

2015

Second

Diabetes

Surgery

Summit.

2000

1970-95

Several reports consistently document remission of diabetes after bariatric surgery.

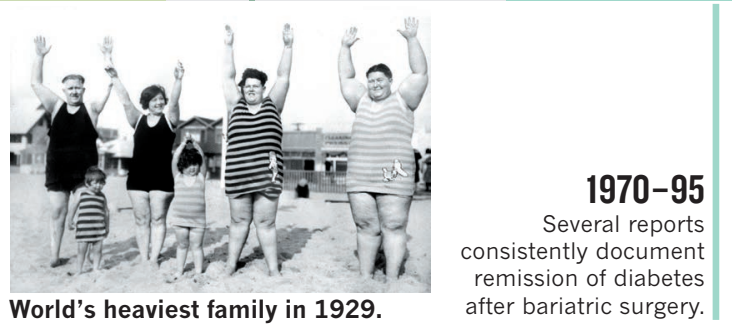

both patients and providers may be encouraged to approach conventional treatments with more determination and rigour.

The broad endorsement of surgery as a treatment option should also inspire fresh approaches in research. Researchers and clinicians are already trying to mimic the effects of gastrointestinal surgery using lessinvasive interventions. For instance, experiments originally conducted in rats, and various studies in humans, have shown that blocking intestinal signalling from the duodenum or upper small intestine can alleviate the symptoms of diabetes ${ }^{6}$.

One approach aims to do this by means of a tube inserted into the intestine. Designed to prevent contact between nutrients and the lining of the upper small intestine, this tube mimics the effects of a surgical bypass. The device has been approved for clinical use in Europe and Australia. Another approach involves passing a balloon-tipped device through the mouth and down into the duodenum, where it is filled with hot water to burn (ablate) the cellular lining. A clinical trial of the device is currently in the

\section{GASTRIC BYPASS}

The stomach is reduced to a small pouch and is connected directly to the intestine. diabetes are rising rapidly in low- and middle-income countries ${ }^{3}$, where surgery is not likely to be available for most patients. But if handled the right way, the inclusion of surgery as an option could influence diabetes care as a whole.

Currently, many people with diabetes and obesity grow disheartened after trying one treatment after another to no avail. Just knowing that through surgery the possibility of major improvement and even remission exists may be empowering to some. Also, to identify those people for whom surgery may be appropriate, providers will first need to be confident that other options have failed ${ }^{1}$. So

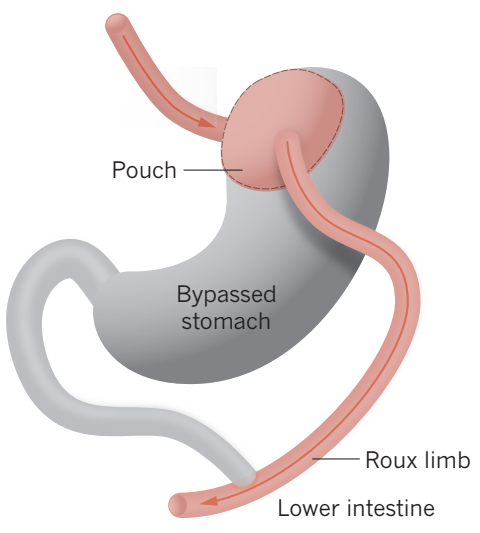

recruitment stage in Europe. Pharmacological interventions that target gastrointestinal mechanisms of metabolic regulation are also being investigated.

The ability of gastrointestinal surgery to influence glucose homeostasis and clinically reverse diabetes suggests that the disease might be explained, at least in part, by a fault in the mechanisms through which the gut regulates metabolism ${ }^{8}$. Testing this hypothesis could provide insights about ways to prevent and even cure diabetes.

Despite the compelling results of clinical trials and experimental work on nutrient-gut signalling mechanisms, shifting diabetes' image as an incurable, hopeless condition caused by excess fat will still require some imagination. Albert Einstein once said that imagination is more important than knowledge. The story of surgery and diabetes shows how important it is to have both.

Francesco Rubino is professor of metabolic and bariatric surgery at King's College London and consultant surgeon at King's College Hospital, London, UK. He is an organizer of the Diabetes Surgery Summit. e-mail:francesco.rubino@kcl.ac.uk

1. Rubino, F. et al. Diabetes Care 39, 861-877 (2016).

2. Leyton, O. Lancet 206, 1162-1163 (1925).

3. NCD Risk Factor Collaboration Lancet 387 , 1513-1530 (2016).

4. Mingrone, G. et al. Lancet 386, 964-973 (2015)

5. Klein, S., Ghosh, A., Cremieux, P. Y., Eapen, S. \& McGavock, T. J. Obesity 19, 581-587 (2011).

6. Rubino, F. \& Marescaux, J. Ann. Surg. 239, 1-11 (2004).

7. Dixon, J. B., Lambert, E. A. \& Lambert, G. W. Mol. Cell. Endocrinol. 418, 143-152 (2015).

8. Rubino, F. Diabetes Care 31, S290-S296 (2008).

9. Pories, W. J. et al. Ann. Surg. 222, 339-350 (1995). 10.Cohen, R. V., Schiavon, C. A., Pinheiro, J. S., Correa, J. L. \& Rubino, F. Surg. Obes. Relat. Dis. 3 , 195-197 (2007).

The author declares competing financial interests: see go.nature.com/49vyhn for details. 